\title{
CLINICOPATHOLOGICAL PARAMETERS RELATED TO MALIGNANT TRANSFORMATION OF ORAL LEUKOPLAKIA: A META-ANALYSIS
}

\begin{abstract}
Objective: To assess the clinical-pathological factors related to the malignant transformation of oral leukoplakia.
\end{abstract}

Materials and Methods: A search for articles on malignant transformation factors related to oral leukoplakia was conducted in the following electronic databases: PubMed (MEDLINE, Cochrane Library), Web of Science (WoS) and Google Scholar. Thirty-seven articles with a low-moderate risk of bias according to the Newcastle-Ottawa methodological quality scale were included in this meta-analysis. The data were analyzed using the statistical programs RevMan 5.4 (The Cochrane Collaboration, Oxford, UK) and MedCalc Statistical Software version 16.4.3 (MedCalc Software Ltd. Ostend, Belgium) programs. The estimated prevalence was calculated according to DerSimonian and Laird random method. For dichotomous outcomes, the estimates of effects of an intervention were expressed as odds ratios (OR) using the Mantel-Haenszel (M-H) method with $95 \%$ confidence intervals.

Results: The estimated global prevalence of malignant transformation of oral leukoplakia was $9.15 \%$. The factors with the highest malignant transformation risk of oral leukoplakia were: non-homogeneous clinical types (OR: 5.41; $\mathrm{p}<0.001$ ); leukoplakias with moderate-severe dysplasia (OR: 3.43; $\mathrm{p}<0.001$ ); lesions located on the tongue and/or the floor of the mouth (OR: 3.19; $\mathrm{p}<0.001)$; leukoplakias in non-smokers (OR: 2.08; $\mathrm{p}<0.001)$ and lesions in women (OR: $1.73 ; \mathrm{p}<0.001)$. In contrast, older age or regular alcohol intake were factors without significant influence $(\mathrm{p}>0.05)$.

Conclusions: Non-homogenous oral leukoplakias and with moderate-severe dysplasia are those with the highest probability of malignant transformation.

Keywords: Cell transformation, neoplastic, epidemiology, leukoplakia oral, risk factors.
*Alberto Rodriguez-Archilla ${ }^{1}$

Cristina Fuentes-Perez ${ }^{2}$

ORCID IDs of the authors:

A.R.A. 0000-0003-1023-943X

C.F.P. $\quad 0000-0002-5764-1525$

${ }^{1}$ Department of Stomatology, Oral Medicine Unit. Faculty of Dentistry. University of Granada, Granada (Spain).

Received : $\quad 04.01 .2021$

Accepted : 01.04 .2021

How to Cite: Rodriguez-Archilla A, Fuentes-Perez C. Clinicopathological Parameters Related to Malignant Transformation of Oral Leukoplakia: A Meta-Analysis. Cumhuriyet Dent J 2021;24:2:197-204.

* Corresponding Author:

Department of Stomatology, Oral Medicine Unit. Faculty of Dentistry. University of Granada. Colegio Maximo, s/n. Campus de Cartuja. 18071-Granada (Spain)

Phone: +34958 244085 E-mail: alberodr@ugr.es 


\section{INTRODUCTION}

In 2005, the World Health Organization (WHO) in collaboration with the UK Center for Oral Cancer and Precancer carried out a redefinition of oral leukoplakia (OL) as a "white plaque of questionable risk whose diagnosis is reached having excluded other known diseases or disorders that do not involve an increased cancer risk". ${ }^{1}$

$\mathrm{OL}$ is the most common potentially malignant disorder of the oral mucosa with an overall prevalence of around $2.6 \% .^{2}$ Clinically, two main clinical forms are differentiated: a) homogeneous, with uniform white lesions and b) nonhomogeneous, within which three types are distinguished: speckled, nodular and verrucous. ${ }^{3}$ Its true potential for malignancy is not yet well established and is under constant analysis. Most oral leukoplakias are related to tobacco and/or alcohol consumption. However, idiopathic leukoplakias not associated with these factors have a higher rate of malignant transformation. According to different studies, the OL malignant transformation rate ranges between $0.13 \%$ and $34 \% .{ }^{4}$ The purpose of this study was to assess the clinicopathological factors related to the malignant transformation of oral leukoplakia.

\section{MATERIALS Y METHODS}

According to the Preferred Reporting Items for
Systematic Reviews and Meta-Analyses (PRISMA) guidelines, a search for studies on risk factors for malignant transformation of oral leukoplakia was performed in the following databases: PubMed (MEDLINE, Cochrane Library), Web of Science (WoS) and Google Scholar. Search strategies included a combination of Medical Subjects Headings (MeSH) and free-text terms. The search terms were as follows: "leukoplakia, oral" [MeSH Terms] AND ("cell transformation, neoplastic" [MeSH Terms] OR "malignant" [All Fields] OR "transformation" [All Fields] OR "degeneration" [All Fields] OR "progression" [All Fields]); "Oral", "leukoplakia" AND "malignant"; allintitle: "malignant", "oral leukoplakia". After this initial search, 2994 articles were found (1029 in PubMed, 1867 in WoS and 98 in Google Scholar), 1792 of them duplicates, leaving 1202 articles for review. Article titles and abstracts were examined independently by two authors (ARA and CFP) that selected jointly the articles to include in the present study. The exclusion criteria were: a) articles without full-text availability ( $\mathrm{n}=563)$; b) articles with a score of fewer than 6 stars out of a maximum of 9 on the Newcastle-Ottawa methodological quality assessment scale $^{5}(\mathrm{n}=134)$, and c) studies with non clinical or non-usable data $(n=468)$. Finally, 37 studies were included in the metaanalysis (Figure 1).

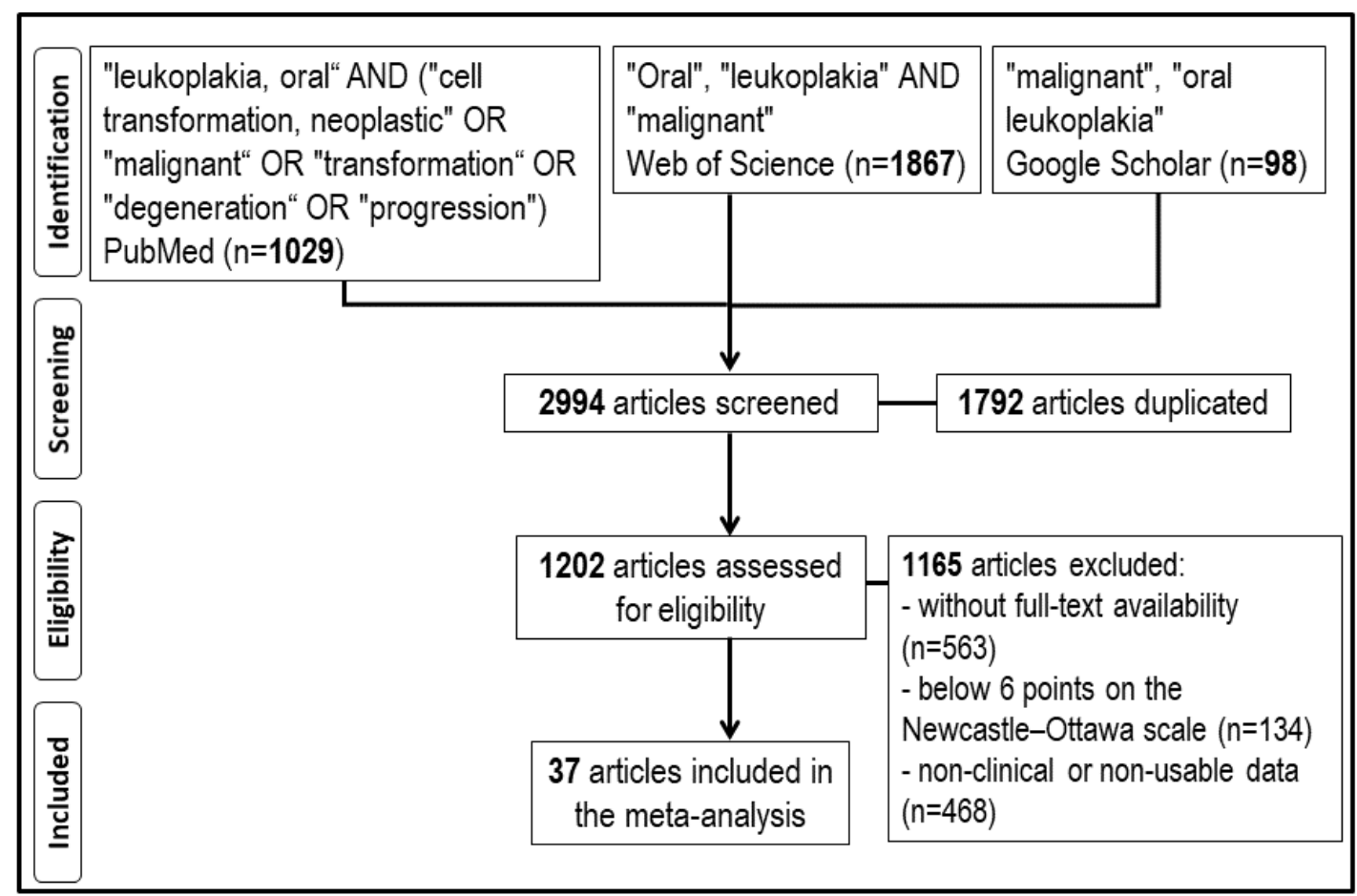

Figure 1. Study flow diagram. 


\section{Statistical Analysis}

Data were statistically analyzed with the RevMan 5.4 (The Cochrane Collaboration, Oxford, UK) and MedCalc Statistical Software version 16.4.3 (MedCalc Software Ltd. Ostend, Belgium) programs. The estimated prevalence was calculated according to DerSimonian and Laird random method. For dichotomous outcomes, the odds ratio (OR) with the Mantel-Haenszel Chi-square formula $(\mathrm{M}-\mathrm{H})$ and $95 \%$ confidence intervals $(95 \% \mathrm{CI})$ was used. Heterogeneity was determined according to the $\mathrm{P}$ values, Cochran's Q, and Higgins statistic $\left(\mathrm{I}^{2}\right)$. A random-effects model was applied if the heterogeneity was high $\left(\mathrm{I}^{2}>50 \%\right)$. The minimum level of significance was set at $\mathrm{p}<0.05$.

\section{RESULTS}

Table 1 presents the thirty-seven studies ${ }^{6-42}$ that analyzed the prevalence of malignant transformation of oral leukoplakia in populations from 15 different countries. The estimated global prevalence of malignant oral leukoplakia was 9.15\% (95\% CI: 6.71 to $11.93 \%$ ) with a range from the maximum prevalence of $34.00 \%$ (95\% CI: 21.20 to $48.76 \%)^{24}$ to the minimum of $0.12 \%$ (95\% CI: 0.04 to $0.27 \%){ }^{10}$

Table 1. Studies on the prevalence (and 95\% CI) of malignant transformation of oral leukoplakia

\begin{tabular}{|c|c|c|c|c|c|}
\hline Study & Year & Country & Sample size & Prevalence & $(95 \% \mathrm{CI})$ \\
\hline Einhorn $^{6}$ & 1967 & Sweden & 782 & $1.53 \%$ & $(0.79$ to $2.66 \%)$ \\
\hline Pindborg $^{7}$ & 1968 & Denmark & 214 & $5.14 \%$ & (2.59 to $9.01 \%)$ \\
\hline Gangadharan $^{8}$ & 1971 & India & 1411 & $4.46 \%$ & (3.44 to $5.67 \%$ ) \\
\hline Roed-Petersen ${ }^{9}$ & 1971 & Denmark & 331 & $2.71 \%$ & (1.25 to $5.09 \%)$ \\
\hline Silverman ${ }^{10}$ & 1976 & India & 4762 & $0.12 \%$ & (0.04 to $0.27 \%)$ \\
\hline Banoczy $^{11}$ & 1977 & Hungary & 670 & $5.97 \%$ & (4.29 to $8.04 \%)$ \\
\hline Kramer $^{12}$ & 1978 & UK & 29 & $24.13 \%$ & (10.29 to $43.54 \%)$ \\
\hline Pogrel $^{13}$ & 1979 & UK & 19 & $15.78 \%$ & (3.38 to $39.57 \%$ ) \\
\hline Gupta $^{14}$ & 1980 & India & 735 & $1.63 \%$ & (0.84 to $2.83 \%)$ \\
\hline Roch-Berry ${ }^{15}$ & 1981 & UK & 117 & $17.09 \%$ & (10.76 to $25.15 \%)$ \\
\hline Silverman ${ }^{16}$ & 1984 & USA & 257 & $17.51 \%$ & (13.06 to $22.71 \%$ ) \\
\hline Lind $^{17}$ & 1987 & Norway & 157 & $8.91 \%$ & (4.96 to $14.50 \%)$ \\
\hline Hogewind $^{18}$ & 1989 & Netherlands & 46 & $6.52 \%$ & (1.36 to $17.89 \%)$ \\
\hline $\mathrm{Shi}^{19}$ & 1992 & China & 235 & $13.61 \%$ & (9.50 to $18.67 \%)$ \\
\hline Schepman ${ }^{20}$ & 1998 & Netherlands & 166 & $12.04 \%$ & (7.51 to $17.99 \%)$ \\
\hline $\mathrm{Ge}^{21}$ & 1999 & China & 211 & $6.63 \%$ & (3.67 to $10.88 \%$ ) \\
\hline Saito $^{22}$ & 1999 & Japan & 111 & $7.20 \%$ & (3.16 to $13.70 \%)$ \\
\hline Guan $^{23}$ & 2001 & China & 110 & $11.81 \%$ & (6.44 to $19.36 \%)$ \\
\hline Napier $^{24}$ & 2003 & UK & 50 & $34.00 \%$ & ( 21.20 to $48.76 \%)$ \\
\hline Mishra $^{25}$ & 2005 & India & 2920 & $0.68 \%$ & (0.41 to $1.05 \%)$ \\
\hline Lee $^{26}$ & 2006 & Taiwan & 1046 & $12.90 \%$ & (10.93 to $15.09 \%)$ \\
\hline $\mathrm{Hsue}^{27}$ & 2007 & Taiwan & 913 & $3.61 \%$ & $(2.50$ to $5.03 \%)$ \\
\hline Arduino ${ }^{28}$ & 2009 & Italy & 207 & $7.24 \%$ & (4.11 to $11.67 \%$ ) \\
\hline $\operatorname{Lan}^{29}$ & 2009 & China & 409 & $12.71 \%$ & (9.64 to $16.33 \%)$ \\
\hline $\mathrm{Zhu}^{30}$ & 2009 & China & 150 & $9.33 \%$ & (5.19 to $15.16 \%)$ \\
\hline $\mathrm{Liu}^{31}$ & 2010 & China & 218 & $17.89 \%$ & (13.04 to $23.63 \%)$ \\
\hline Wang $^{32}$ & 2011 & China & 576 & $11.45 \%$ & (8.97 to $14.34 \%)$ \\
\hline Warnakulasuriya $^{33}$ & 2011 & UK & 335 & $6.86 \%$ & (4.40 to $10.12 \%)$ \\
\hline Brzak $^{34}$ & 2012 & Croatia & 54 & $1.85 \%$ & (0.04 to $9.89 \%)$ \\
\hline $\mathrm{Gao}^{35}$ & 2012 & China & 1832 & $4.64 \%$ & (3.72 to $5.70 \%)$ \\
\hline $\mathrm{Ho}^{36}$ & 2012 & UK & 91 & $25.27 \%$ & (16.74 to $35.47 \%)$ \\
\hline $\mathrm{Liu}^{37}$ & 2012 & China & 320 & $17.81 \%$ & (13.77 to $22.45 \%$ ) \\
\hline $\operatorname{Lian}^{38}$ & 2013 & Taiwan & 1238 & $7.10 \%$ & (5.74 to $8.68 \%)$ \\
\hline
\end{tabular}




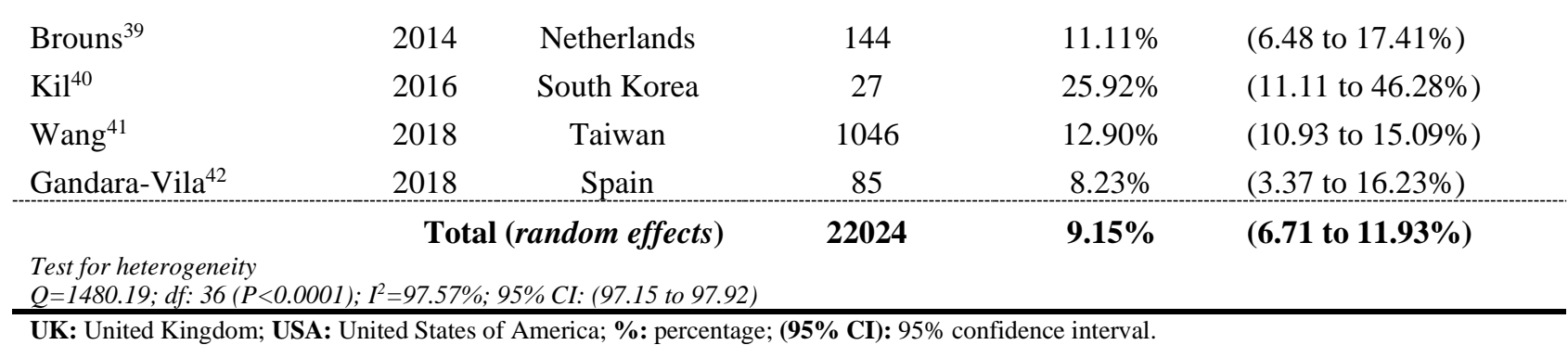

UK: United Kingdom; USA: United States of America; \%: percentage; (95\% CI): 95\% confidence interval.

The main risk factors related to the malignant

transformation of oral leukoplakia are shown in Table 2.

Table 2. Main risk factors related to malignant transformation of oral leukoplakia (OL)

\begin{tabular}{lclcccc}
\hline Risk factor & $\mathbf{n}$ & Outcome & OR & (95\% CI) & $\mathbf{I}^{\mathbf{2}}$ & p-value \\
\hline Age & 6 & $>60$ years-old & 1.56 & $(0.95$ to 2.57$)$ & $46 \%$ & 0.08 \\
Gender & 22 & Female & 1.73 & $(1.47$ to 2.05$)$ & $21 \%$ & $<0.001 *$ \\
OL clinical type & 13 & Non-homogeneous & 5.41 & $(3.24$ to 9.02$)$ & $61 \%$ & $<0.001 *$ \\
Degree of dysplasia & 6 & Moderate-Severe & 3.43 & $(2.30$ to 5.11$)$ & $0 \%$ & $<0.001 *$ \\
OL Location & 9 & Tongue- FM & 3.19 & $(2.26$ to 4.50$)$ & $41 \%$ & $<0.001 *$ \\
Smoking & 6 & Non-smoker & 2.08 & $(1.51$ to 2.80$)$ & $0 \%$ & $<0.001 *$ \\
Drinking & 6 & Non-drinker & 1.02 & $(0.76$ to 1.38$)$ & $0 \%$ & 0.87 \\
\hline
\end{tabular}

n: number of studies; OR: Odds Ratio; (95\% CI): 95\% confidence interval; I' $\mathbf{I}^{2}$ Higgins statistic for heterogeneity (percentage); FM: floor of the mouth; *statistically significant.

Six studies ${ }^{11,28,31,33,37,42}$ examined the possible influence of age on the oral leukoplakia (OL) malignant transformation risk. Although the malignant transformation of the oral leukoplakia was more frequent in those older than 60 years, age was a factor without influence on the OL malignancy. After statistical analysis, no significant relationship was observed $(\mathrm{OR}=1.56$; $95 \%$ CI: 0.95 to $2.57 ; \mathrm{p}=0.08$ ).

Twenty-two studies $7,9-11,13,15-20,24,26,28-33,35,37,40$ considered gender as a possible OL malignant transformation risk factor. Women were 1.73 times more likely to present malignant leukoplakias compared to men, finding a highly significant statistical association (OR=1.73; 95\% CI: 1.47 to 2.05; $\mathrm{p}<0.001)$.

Thirteen studies ${ }^{11,14,17-19,24-26,28-30,36,37}$ assessed the clinical type of oral leukoplakia (OL). Nonhomogeneous OL had a 5.41-fold increase in the risk of malignant transformation compared to homogeneous ones, with highly significant statistical differences (OR=5.41; 98\% CI: 3.24 to 9.02; $\mathrm{p}<0.001)$.

Six studies ${ }^{20,28,33,36,37,43}$ analyzed the degree of epithelial dysplasia of oral leukoplakias (OLs). Moderate-severe dysplasia OLs were 3.43 times more likely to malignant transformation than mild dysplasia OLs. After statistical analysis, a highly significant relationship was found $(\mathrm{OR}=3.43 ; 95 \%$ CI: 2.30 to $5.11 ; \mathrm{p}<0.001)$.

Nine studies ${ }^{11,20,25,26,28,31,37,39,42}$ determined the oral leukoplakias malignant transformation risk based on the location of the lesions. Oral leukoplakias located on the tongue and/or floor of the mouth increased 3.19 times the risk of malignant transformation with a highly significant statistical association (OR=3.19; 95\% CI: 2.26 to $4.50 ; \mathrm{p}<0.001)$.

Six studies ${ }^{26,28,31,37,39,42}$ evaluated the role of harmful habits, both tobacco consumption and alcohol intake, on the malignant transformation risk of oral leukoplakia (OL). Non-smoking patients with oral leukoplakias had 2.08 times more risk, observing highly significant statistical differences $\quad(\mathrm{OR}=2.08 ; 95 \%$ CI: 1.54 to 2.80 ; $\mathrm{p}<0.001)$. The alcohol intake had no significant effect on the OL malignant transformation probability $(\mathrm{OR}=1.02 ; 95 \% \mathrm{CI}: 0.76$ to 1.38 ; $\mathrm{p}=0.87$ ).

\section{DISCUSSION}

Data from thirty-seven studies on the clinicopathological factors related to malignant 
transformation of oral leukoplakia have been considered in the present meta-analysis.

In this paper, the estimated global prevalence of malignant transformation of oral leukoplakia was $9.15 \% \quad(95 \% \quad \mathrm{CI}: \quad 6.71$ to $11.93 \%)$. Warnakulasuriya et al. ${ }^{4}$ conducted a systematic review of 24 studies in which they analyzed the rate of malignant transformation of oral leukoplakias, finding 405 malignant cases (3.5\%) out of a total of 11423 leukoplakias, a prevalence rate lower than that observed in our study.

In the present study, an older age did not imply a greater risk of malignant transformation of oral leukoplakias, without a statistically significant relationship $(\mathrm{p}=0.08)$. Of the six studies that examined age, five of them ${ }^{11,28,31,33,37}$ observed more malignant lesions in patients older than 60 years and only one,${ }^{42}$ in those younger than this age. The findings of the different studies on the role of age were highly variable. Some researchers argue that age is not a risk factor, ${ }^{31}$ while others state that the patient's age is an important risk factor that affects malignant transformation and that it may be correlated with individual genetic susceptibility. ${ }^{37}$ There is no clear evidence that older age increases the probability of malignant progression in oral leukoplakia. Older people, probably due to immune dysfunction and long-term exposure to other associated risk factors, may be more susceptible to malignancy. ${ }^{4}$

In the present study, women were 1.73 times more likely to present malignant oral leukoplakias with a highly significant statistical association ( $\mathrm{p}<0.001)$. Of the 22 studies that considered gender, nineteen ${ }^{7,9-11,16-20,24,26,29-33,35,37,40}$ agreed to observe more malignant lesions in women; meanwhile, three of them ${ }^{13,15,28}$ observed it more in men. Most of these studies indicate that females' oral leukoplakias have a higher risk of malignant transformation. Although oral leukoplakia is relatively uncommon in women compared to men, the malignant transformation of leukoplakia is significantly higher in women. The reason why females are more predisposed to the malignant transformation of its lesions remains a paradox. ${ }^{4}$ Moreover, an increased risk of malignant transformation of oral leukoplakia has been observed in non-smoking women. It is not clear either why the absence of smoking habit is associated with an increased risk of malignant transformation only in women. ${ }^{20}$

In this study, non-homogeneous oral leukoplakias increased 5.41-fold their risk of malignancy, observing highly significant statistical differences $(p<0.001)$. Twelve studies ${ }^{11,14,17,19,24-}$ 26,28-30,36,37 pointed out a higher probability of malignancy in non-homogeneous oral leukoplakias. Similarly, a higher degree of dysplasia (moderate-severe) raised 3.43-fold the probability of malignancy, with a highly significant relationship $(\mathrm{p}<0.001)$. Of the 6 studies that examined the degree of dysplasia, five of them ${ }^{20,28,33,36,37}$ showed a higher risk of malignancy in the most dysplastic leukoplakias and only one ${ }^{43}$ did not observe this fact, without statistically significant results. Almost all studies claim that non-homogeneous oral leukoplakias show a higher malignant transformation risk compared to homogeneous leukoplakias that appear to follow a more benign course. In non-homogeneous lesions, it is important an adequate choice of the biopsy site since it will condition its correct diagnosis, as well as a more aggressive treatment for this type of leukoplakias. Lee et al. ${ }^{26}$ in their study of 1046 oral leukoplakia found that non-homogeneous lesions were 5.69 times more likely to present epithelial dysplasia and 28.13 times more likely to malignant transformation. $72.6 \%$ of the non-homogeneous leukoplakias and $33.3 \%$ of the homogeneous ones were dysplastic lesions. Malignant transformation of non-homogeneous oral leukoplakias approached $20 \%$ in contrast to less than $1 \%$ in homogeneous leukoplakias. Classifying the grade of dysplasia as: no dysplasia, mild, moderate, and severe, lends itself to some subjectivity in interpretation by pathologists and could distort the results. Therefore, in an attempt to unify criteria, a classification into two categories has been proposed: a) no/questionable/mild - low-grade dysplasia and b) moderate/severe - high-grade dysplasia). High-grade dysplasias are closely associated with an increased malignant transformation risk and in shorter periods. This justifies periodic follow-up during the first 2-3 
years for patients with high-grade dysplastic oral leukoplakias to be able to detect early events of malignant transformation. ${ }^{37}$

In this study, oral leukoplakias located on the tongue and/or floor of the mouth were 3.19 times more likely to suffer malignant transformation with a highly significant statistical association ( $\mathrm{p}<0.001)$. The nine studies ${ }^{11,20,25,26,28,31,37,39,42}$ that focused on the location of oral leukoplakia, showed that the lingual location and/or on the floor of the mouth were the ones with the highest risk of malignant transformation. In a systematic review, the most frequent locations of malignant oral leukoplakias were the tongue $(24.22 \%$ of cases) and the floor of the mouth (14.85\%). ${ }^{4}$ Lee et al. ${ }^{26}$ found that leukoplakias located on the tongue and/or the floor of the mouth increased 1.84 times the probability of epithelial dysplasia and 2.72 times the risk of malignancy compared to leukoplakias with other locations. Severe dysplastic lesions tended to be located on the ventrolateral lingual surface and the floor of the mouth, showing greater potential for malignant transformation and a greater frequency of loss of heterozygosity in chromosomes $3 p, 9 p$ and $17 p$. A possible explanation for this higher risk in these locations would be that these areas are substantially more exposed to local carcinogens due to the greater permeability of the oral mucosa. ${ }^{42}$

In the present investigation, surprisingly nonsmokers had an increase of 2.08 times the malignant transformation risk of their leukoplakias with highly significant statistical differences ( $\mathrm{p}<0.001)$. Regular alcohol consumption did not have a significant impact on the malignancy of oral leukoplakia, without reaching statistical significance $\quad(p=0.87)$. All studies ${ }^{26,28,31,37,39,42}$ indicated an increased malignant risk in leukoplakias from non-smoking subjects. In the case of alcohol intake, three of them ${ }^{26,37,42}$ found a higher malignant transformation risk in lesions from non-drinkers, compared to another three ${ }^{28,31,39}$ who did show it in lesions from drinkers. However, none of the results of these studies was statistically significant. The tobacco and/or alcohol consumption play an important role in the development of oral leukoplakia, although their role in its malignant transformation remains controversial and it has not been clarified yet. ${ }^{37}$ Smoking and drinking are well-established risk factors for dysplastic oral leukoplakia and oral cancer, but not for the malignant transformation risk from leukoplakia. Various studies show that smoking-related oral leukoplakias appear to have less malignant potential than those not associated with smoking. The reasons for these seemingly paradoxical findings remain unclear. Several possible explanations could be raised: 1) in the absence of tobacco and/or alcohol as stimulating agents, there are other more potent carcinogenic factors; 2) since carcinogenesis is a multistep process, the factors that condition the development of oral leukoplakias would be different from the factors responsible for the malignant transformation of these lesions; 3) the lower malignant transformation risk of leukoplakias among smokers/drinkers could be justified in that the oral carcinogenesis process is more prolonged in non-smokers/abstainers and, therefore, the evolution time of oral leukoplakias is longer predisposing them to malignancy. ${ }^{26}$

This study has some limitations. The periods of follow-up of the lesions are highly variable in the different studies. In some of them, they are too short and there is probably an underestimation of cases of malignancy. Considering the harmful habits, on some occasions, the quantity and age of the habit, as well as the alcohol content of the beverages, could not be adequately evaluated. The influence of superinfection of oral leukoplakias by human papillomavirus (HPV) and/or Candida species could not be analyzed either.

Finally, the high heterogeneity observed in some comparisons of this meta-analysis demands a cautious interpretation.

\section{CONCLUSIONS}

In this study, the estimated global prevalence of malignant transformation of oral leukoplakia was $9.15 \%$. The factors with the highest malignant transformation risk of oral leukoplakia were: nonhomogeneous clinical types (OR:5.41; $<<0.001$ ); leukoplakias with moderate-severe dysplasia (OR:3.43; $\mathrm{p}<0.001$ ); lesions located on the tongue 
and/or the floor of the mouth (OR:3.19; $<<0.001)$; leukoplakias in non-smokers (OR:2.08; $\mathrm{p}<0.001)$ and lesions in women (OR:1.73; $\mathrm{p}<0.001)$. In contrast, older age or regular alcohol intake were factors without significant influence ( $p>0.05)$.

\section{CONFLICTS OF INTEREST STATEMENT}

No conflicts of interest.

\section{REFERENCES}

1. Warnakulasuriya S, Johnson NW, van der Waal I. Nomenclature and classification of potentially malignant disorders of the oral mucosa. J Oral Pathol Med. 2007;36:575-580.

2. Ganesh D, Sreenivasan P, Öhman J, Wallström M, Braz-Silva PH, Giglio D, Kjeller G, Hasséus B. Potentially Malignant Oral Disorders and Cancer Transformation. Anticancer Res. 2018;38:3223-3229.

3. Arduino PG, Bagan J, El-Naggar AK, Carrozzo M. Urban legends series: oral leukoplakia. Oral Dis. 2013;19:642-659.

4. Warnakulasuriya S, Ariyawardana A. Malignant transformation of oral leukoplakia: a systematic review of observational studies. J Oral Pathol Med. 2016;45:155-166.

5. Wells G, Shea B, O'Connell D, Peterson J, Welch V, Losos M, Tugwell P. The Newcastle-Ottawa Scale (NOS) for assessing the quality of nonrandomised studies in meta-analyses [Internet]. Ottawa (Canada): The Ottawa Hospital. Available from: http://www.ohri.ca/programs/clinical_epidemiology/ox ford.asp.

6. Einhorn J, Wersall J. Incidence of oral carcinoma in patients with leukoplakia of the oral mucosa. Cancer. 1967;20:2189-2193.

7. Pindborg JJ, Jolst O, Renstrup G, Roed-Petersen B. Studies in oral leukoplakia: a preliminary report on the period pervalence of malignant transformation in leukoplakia based on a follow-up study of 248 patients. J Am Dent Assoc. 1968;76:767-771.

8. Gangadharan P, Paymaster JC. Leukoplakia--an epidemiologic study of 1504 cases observed at the Tata Memorial Hospital, Bombay, India. Br J Cancer. 1971;25:657-668.

9. Roed-Petersen B. Cancer development in oral leukoplakia follow-up of 331 patients. J Dent Res. 1971; 50:711-712.

10. Silverman S, Bhargava K, Smith LW, Malaowalla AM. Malignant transformation and natural history of oral leukoplakia in 57,518 industrial workers of Gujarat, India. Cancer. 1976;38:1790-1795.

11. Bánóczy J. Follow-up studies in oral leukoplakia. J Maxillofac Surg. 1977;5:69-75.

12. Kramer IR, El-Labban N, Lee KW. The clinical features and risk of malignant transformation in sublingual keratosis. Br Dent J. 1978;144:171-180.

13. Pogrel MA. Sublingual keratosis and malignant transformation. J Oral Pathol. 1979;8:176-178.

14. Gupta PC, Mehta FS, Daftary DK, et al. Incidence rates of oral cancer and natural history of oral precancerous lesions in a 10-year follow-up study of Indian villagers. Community Dent Oral Epidemiol. 1980;8:283-333.

15. Roch-Berry CS. Malignant changes in glossal leukoplakia. Clin Radiol. 1981;32:693-694.

16. Silverman S Jr, Gorsky M, Lozada F. Oral leukoplakia and malignant transformation. A follow-up study of 257 patients. Cancer. 1984;53:563-568.

17. Lind PO. Malignant transformation in oral leukoplakia. Scand J Dent Res. 1987;95:449-455.

18. Hogewind WF, van der Kwast WA, van der Waal I. Oral leukoplakia, with emphasis on malignant transformation. A follow-up study of 46 patients. J Craniomaxillofac Surg. 1989;17:128-133.

19. Shi HB. Analysis of malignancy change of oral leukoplakia. Shanghai Kou Qiang Yi Xue. 1992;1:63-5. 20. Schepman KP, van der Meij EH, Smeele LE, van der Waal I. Malignant transformation of oral leukoplakia: a follow-up study of a hospital-based population of 166 patients with oral leukoplakia from The Netherlands. Oral Oncol. 1998;34:270-275.

21. Ge HB, Sun Z, Shen SL. A clinical analysis of 211 oral leukoplakia. Beijing J Stomatol. 1999;3:11-12.

22. Saito T, Sugiura C, Hirai A, et al. High malignant transformation rate of widespread multiple oral leukoplakias. Oral Dis. 1999;5:15-19.

23. Guan XB, Sun Z, Wang JB. Preliminary study on the relationship between smoking, malignant transformation of oral leukoplakia. Beijing J Stomatol. 2001;3:123-124.

24. Napier SS, Cowan CG, Gregg TA, Stevenson M, Lamey PJ, Toner PG. Potentially malignant oral lesions in Northern Ireland: size (extent) matters. Oral Dis. 2003;9:129-137.

25. Mishra M, Mohanty J, Sengupta S, Tripathy S. Epidemiological and clinicopathological study of oral 
leukoplakia. Indian J Dermatol Venereol Leprol. 2005;71:161-165.

26. Lee JJ, Hung HC, Cheng SJ, et al. Carcinoma and dysplasia in oral leukoplakias in Taiwan: prevalence and risk factors. Oral Surg Oral Med Oral Pathol Oral Radiol Endod. 2006;101:472-480.

27. Hsue SS, Wang WC, Chen CH, Lin CC, Chen YK, Lin LM. Malignant transformation in 1458 patients with potentially malignant oral mucosal disorders: a followup study based in a Taiwanese hospital. J Oral Pathol Med. 2007;36:25-29.

28. Arduino PG, Surace A, Carbone M, et al. Outcome of oral dysplasia: a retrospective hospital-based study of 207 patients with a long follow-up. J Oral Pathol Med. 2009;38:540-544.

29. Lan AX, Guan XB, Sun Z. Analysis of risk factors for carcinogenesis of oral leukoplakia. Zhonghua Kou Qiang Yi Xue Za Zhi. 2009;44:327-331.

30.Zhu QS, Miao QA, Zhi KQ, Wang YC. A clinical analysis of 150 oral leukoplakia abstract. J Clin Stomatol. 2009;5:311-312.

31. Liu W, Wang YF, Zhou HW, Shi P, Zhou ZT, Tang GY. Malignant transformation of oral leukoplakia: a retrospective cohort study of 218 Chinese patients. BMC Cancer. 2010;10:685.

32. Wang YF, Shang S, Zhou ZT, Tang GY. A retrospective analysis on the malignant transformation rate, time and risk factors of oral leukoplakia. Shanghai Kou Qiang Yi Xue. 2011;20:55-61.

33. Warnakulasuriya $\mathrm{S}$, Kovacevic $\mathrm{T}$, Madden $\mathrm{P}$, et al. Factors predicting malignant transformation in oral potentially malignant disorders among patients accrued over a 10-year period in South East England. J Oral Pathol Med. 2011;40:677-683.

34. Brzak BL, Mravak-Stipetić M, Canjuga I, et al. The frequency and malignant transformation rate of oral lichen planus and leukoplakia--a retrospective study. Coll Antropol. 2012;36:773-777.

35. Gao Y, Guo ZL, Luo HY, Wang J. Clinicopathological characteristics of malignant transformation in 85 cases of oral leukoplakia. Zhonghua Kou Qiang Yi Xue Za Zhi. 2012;47:410-413. 36. Ho MW, Risk JM, Woolgar JA, et al. The clinical determinants of malignant transformation in oral epithelial dysplasia. Oral Oncol. 2012;48(10):969-976.

37. Liu W, Shi LJ, Wu L, et al. Oral cancer development in patients with leukoplakia--clinicopathological factors affecting outcome. PLoS One. 2012;7:e34773.

38. Lian IeB, Tseng YT, Su CC, Tsai KY. Progression of precancerous lesions to oral cancer: results based on the Taiwan National Health Insurance Database. Oral Oncol. 2013;49:427-430.

39. Brouns E, Baart J, Karagozoglu KH, Aartman I, Bloemena E, van der Waal I. Malignant transformation of oral leukoplakia in a well-defined cohort of 144 patients. Oral Dis. 2014;20:e19-24.

40. Kil TJ, Kim HS, Kim HJ, Nam W, Cha IH. Genetic Abnormalities in Oral Leukoplakia and Oral Cancer Progression. Asian Pac J Cancer Prev. 2016;17:3001-6. 41. Wang TY, Chiu YW, Chen YT, et al. Malignant transformation of Taiwanese patients with oral leukoplakia: A nationwide population-based retrospective cohort study. J Formos Med Assoc. 2018;117:374-380.

42. Gandara-Vila P, Perez-Sayans M, Suarez-Penaranda JM, et al. Survival study of leukoplakia malignant transformation in a region of northern Spain. Med Oral Patol Oral Cir Bucal. 2018;23:e413-e420.

43. Holmstrup P, Vedtofte P, Reibel J, Stoltze K. Longterm treatment outcome of oral premalignant lesions. Oral Oncol. 2006;42:461-474. 